\title{
Diagnosis and Treatment of Oesophageal Carcinoma: Changes in Every Respect
}

\author{
Christian Ell ${ }^{a} \quad$ Dietmar Lorenz ${ }^{b}$ \\ a Department of Medicine II/IV, Sana Klinikum Offenbach, Offenbach, Germany, \\ ${ }^{b}$ Department of General and Visceral Surgery, Sana Klinikum Offenbach, Offenbach, Germany
}

In the last decade, no other tumour entity was subject to such major changes in the diagnosis and treatment as oesophageal carcinoma: More and more early cancers are now being discovered with modern methods of endoscopy and by means of the clinical symptom of heartburn. Endosonography has become the most important method for preoperative lymph node assessment. Positron emission tomography-computed tomography (PET-CT) is on the verge of reaching clinical relevance.

Not least due to the work of our Wiesbaden study group endoscopic resection of mucosal carcinomas is now included in the new German guideline for oesophageal cancer as the method of choice while surgical resection was downgraded to second choice treatment. The Union for International Cancer Control (UICC) standards for stages II and III suggest multimodal treatment with perio- perative chemotherapy or neoadjuvant radiochemotherapy, which are associated with a distinct increase of definitive cure rates. In addition, surgical resection was effectively improved in terms of complication rates and postoperative mobilisation by the introduction of minimally invasive laparoscopic as well as thoracoscopic techniques.

If centralisation of oesophagectomy in other countries were enforced similar to The Netherlands, mortality and morbidity could be halved. In this respect, this special issue of ViszeraLMEDIZIN also serves as a call for presenting patients with both early and advanced oesophageal carcinoma in designated centres which have the full range of diagnostic and therapeutic procedures as well as the relevant experience at their disposal.

\section{KARGER}

